\title{
Senescence surveillance maintains genomic and functional differentiation in pre-malignant tumors
}

Thomas Wieder ${ }^{1}$, Ellen Brenner ${ }^{1}$, Nadine Simon ${ }^{1}$, Jürgen Bauer ${ }^{1}$, Felicia Gerst ${ }^{2}$, Susanne Ullrich ${ }^{2}$, Birgit Fehrenbacher ${ }^{1}$, Heidi Braumüller ${ }^{1}$, and Martin Röcken ${ }^{1}$

${ }^{1}$ Department of Dermatology, University Medical Center Tübingen, Eberhard-KarlsUniversity, Liebermeister Strasse 25, 72076 Tübingen, Germany

2Department of Internal Medicine IV, Endocrinology, Diabetology and Clinical Chemistry, Eberhard Karls University, Otfried-Müller-Strasse 10, 72076 Tübingen, Germany

Introduction Different animal models as well as histological studies using samples from cancer patients clearly showed that cellular senescence, in particular cytokine-induced senescence (CIS), is a barrier against tumor progression (1-3). Yet, recent data question this concept suggesting that senescent cells may also promote tumor progression $(4,5)$. By adopting a senescence-associated secretory phenotype (SASP), senescence signaling may even induce dedifferentiation and stemness (5). To shine more light on this controversial question, we investigated the effects of CIS on tumor progression and differentiation. For this, we used pre-malignant $\beta$-cancers from RIP-Tag2 mice that express three differentiation antigens with physiological functions: synaptophysin, insulin and glucose transporter-2 (Glut-2), and treated the mice with Tag-specific $\mathrm{T}$ helper $1\left(T_{H} 1\right)$ cells to induce CIS in vivo (Fig. 1).

Results First, we analyzed the genomic stability of the RIP-Tag2 cancers by comparative genomic hybridization (CGH). We show that $T_{H} 1$-cell-mediated immunity prevented the transition of precancerous adenomas into $\beta$-cancers by inhibition of tumor growth and stabilization their genome. Thus, CIS stopped proliferation (Fig. 2, left) and simultaneously prevented major genomic aberrations, i.e. chromosomal amplifications and losses that repeatedly occurred in RIP-Tag2 cancers of sham-treated Tag2 cancers, we set up an immunofluorescence method to analyze the expression of the differentiation antigens. Tumor progression caused a gradual loss of the functionally relevant differentiation markers insulin and Glut-2 whereas synaptophysin remained constant (Fig. 3, left). Importantly, CIS prevented the loss of insulin and Glut-2 in vivo (Fig. 3, right). mice (Fig. 2, right). To study the differentiation status of RIP-

To demonstrate that the $T_{H}$ 1-cell mediated effects on the differentiation state of RIP-Tag2 cancer cells are mediated by the $\mathrm{T}_{\mathrm{H}} 1$ cell cytokines interferon- $\gamma(\mathrm{IFN}-\gamma)$ and tumor necrosis factor (TNF), we treated isolated $\beta$-cancer cells with recombinant cytokines in vitro. Longterm treatment of RIP-Tag2 cancer cells with a cytokine cocktail of IFN- $\gamma$ and TNF indeed retained the expression of insulin and Glut-2 in vitro (Fig. 4) thereby mimicking the effect of $\mathrm{T}_{\mathrm{H}} 1$ cells in vivo.

Conclusion Our data clearly show that immunity-induced senescence surveillance protects against dedifferentiation and loss of physiological function during development of endocrine tumors.
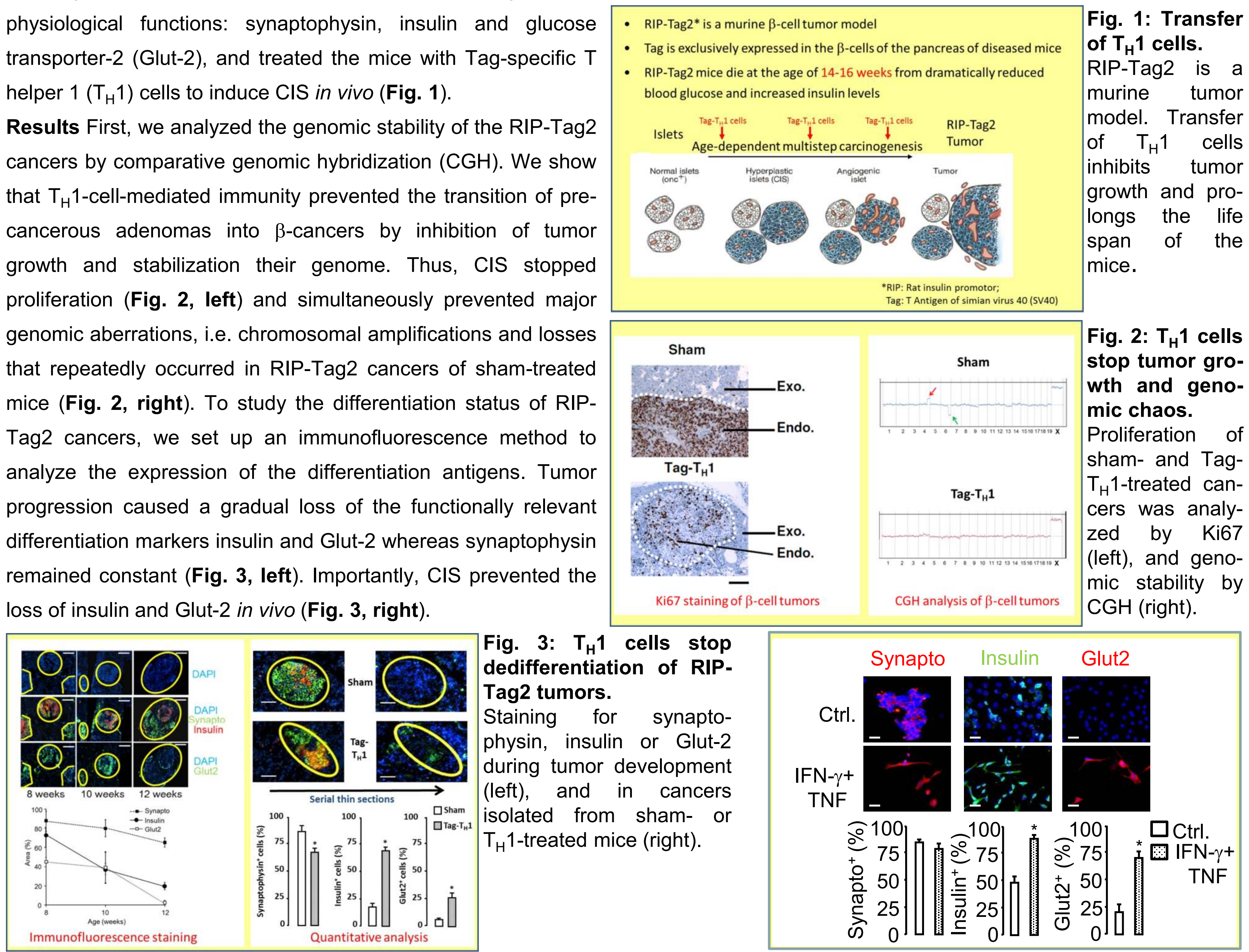

Fig. 3: $T_{H} 1$ cells stop dedifferentiation of RIP. Tag2 tumors.

Staining for synaptophysin, insulin or Glut-2 during tumor development (left), and in cancers isolated from sham- or $\mathrm{T}_{\mathrm{H}}$ 1-treated mice (right).

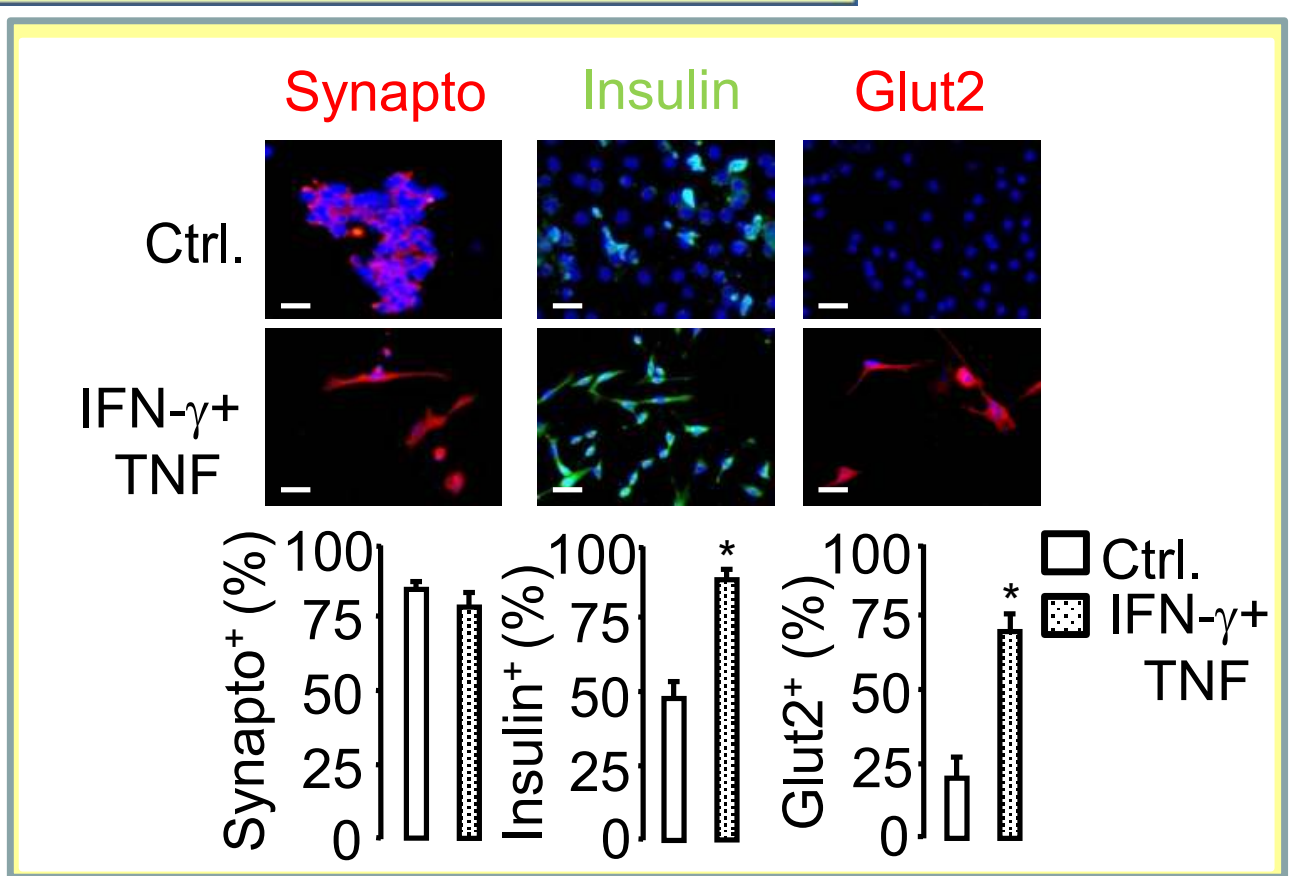

Fig. 4: IFN- $\gamma$ and TNF keep $\beta$-cancer cells in a differentiated state. Staining for synaptophysin, insulin or Glut-2, and quantitative analysis of medium (Ctrl.) or IFN- $\gamma+$ TNF-treated $\beta$-cancer cells. 